\title{
coress feedback
}

\section{CORESS feedback}

This series of reports warns of topical adverse effects of alcoholic skin preparations. Two separate cases involving colorectal malignancies flag up potential for miscommunication of important information at multidisciplinary team meetings, and the need for careful patient selection and staging of patients for transanal rectal surgery. Finally, a 'never event' is described, in which cumulative system failures contributed to the adverse outcome.

We are grateful to those who have provided the material for these reports. The online reporting form is on our website (www.coress.org.uk), which also includes all previous Feedback reports. Published cases will be acknowledged by a 'Certificate of Contribution', which may be included in the contributor's record of continuing professional development.

\section{Eye irritation caused by alcoholic chlorhexidine skin preparation}

(Ref 219)

I had trained my registrar to perform percutaneous glycerol injections for trigeminal neuralgia. After performing this successfully, he inadvertently cleaned the surface landmarks running to the lower eyelid with alcoholic chlorhexidine, which came into contact with the cornea. The eye was washed thoroughly but the patient awoke with an inflamed conjunctiva. She was referred to the ophthalmology team, who prescribed topical antibiotics. The patient made a complete recovery.

\section{Reporter's comments}

There was poor attention to detail. Not enough emphasis during training is placed on the hazards of alcoholic preparations coming into contact with the cornea. After initial skin preparation around the cheek, alcoholic preparations should be removed. Water or saline should be available to clean the skin postoperatively.

\section{CORESS comments}

Alcoholic chlorhexidine may cause irreversible corneal damage when used for facial skin preparation and has also caused sensorineural deafness (secondary to cochlea injury) when used in ear surgery. Cleansing with the preparation should be avoided in these regions. Where exposure has occurred, the preparation should be washed away with water.

\section{MDT mishap}

(Ref 222)

A 42-year-old man with multiple sebaceous adenomas was referred to a medical geneticist who diagnosed Muir-Torre syndrome (a variant of hereditary non-polyposis colorectal cancer). An experienced surgeon set up a surveillance protocol for upper gastrointestinal, colonic and genitourinary surveillance.

A right colon carcinoma was diagnosed ten years later on colonoscopy. Computed tomography (CT) staging revealed a $2.1 \mathrm{~cm}$ coeliac lymph node. The colorectal multidisciplinary team (MDT) noted this report and arranged for positron emission tomography (PET), which showed: '... focal intense uptake in the stomach and coeliac node. This could represent a separate gastric primary malignancy.' Gastroscopy was performed but biopsies were negative.

The patient proceeded to laparoscopic right hemicolectomy. No mention was made on the consent form or operation note about excision biopsy of the coeliac lymph node.

Reported histology of the caecal carcinoma was $\mathrm{pT} 3 \mathrm{pN} 0$. None of the nine resected nodes showed any evidence of metastatic spread.

The postoperative colorectal MDT made no reference to the preoperative PET and summarised: 'no plans for chemotherapy, discharge from colorectal MDT'. CT/PET 12 months later indicated that the coeliac node had increased to $29 \mathrm{~mm}$ in size. Gastroscopy at this time revealed an oesophagogastric carcinoma primary, with histology confirming moderate differentiation. Laparoscopy preoperatively showed dense adhesions but no evidence of intra-abdominal coelomic disease. The patient was treated with neoadjuvant chemotherapy for four months and a gastrectomy was then performed. Stomach histology demonstrated a moderately differentiated yT4 N1 lesion with 4/17 positive lymph nodes. The patient subsequently developed disseminated intra-abdominal metastatic disease.

\section{Reporter's comments}

The case illustrates adverse potential consequences of subdivision of surgical responsibility for intra-abdominal malignant disease. The gastric carcinoma was clearly present when the caecal lesion was diagnosed. The PET demonstrated the coeliac node but no thought was given to excising this at the laparoscopic colonic procedure. The fact that the patient had Muir-Torre syndrome was overlooked. The absence of any positive locoregional colonic nodes, but a PET positive coeliac node, was ignored by a teaching hospital MDT. 
Despite the two-year delay in performing gastrectomy for nodal disease, the patient survived a further two years. Gastrectomy performed at the earliest opportunity following his right hemicolectomy might have been curative.

\section{CORESS comments}

This case highlights the importance of communication between MDTs. There is a real risk of MDTs concentrating solely on the specialty area of interest and failing to consider the patient as a whole. A clinician with knowledge of the patient should be present at all MDT meetings. There was no clear plan for surveillance or biopsy of the coeliac node and the issue of lack of a clinician with overall responsibility for the patient is also raised.

\section{Retained gallbladder at laparoscopic cholecystec- tomy

A 38 year-old patient underwent an urgent laparoscopic cholecystectomy for cholecystitis, the third similar case on a busy theatre list. The operation proceeded uneventfully. The gallbladder was placed in a bag and stowed above the liver prior to a final check of the abdomen before removing the gallbladder. At this point, I noticed some bleeding from the gallbladder fossa, necessitating application of pressure with a tonsil swab, inserted through one of the ports. The swab became soaked with blood, expanding, and it took some effort to extract it through the port. As the swab was delivered, the consultant (who had helped to remove the swab) and I were both relieved to have extracted it. We immediately took the laparoscopic ports out, forgetting that we had not yet extracted the gallbladder, which was still in the bag above the liver.

The situation was compounded by the scrub nurse failing to realise that she had not handed the gallbladder out of the operative field and that the laparoscopic bag had not been counted out of the abdomen. There was also a failure to check whether the specimen was actually in the pot (despite labels being dutifully checked for the surgical safety checklist sign out). The patient was woken up and taken to recovery, and it was only when the pathology department rang to alert the theatre that an empty pot had been received that the mistake was uncovered. The patient was informed immediately and was taken back to theatre from the recovery area, requiring a second anaesthetic to extract the gallbladder in the bag. The team had a full debrief at the end of the day and later participated in root cause analysis.

\section{CORESS and reporter's comments}

This 'never event' demonstrated system errors involving the whole team. Distraction occurred at a critical point in the operation and focus on the task in hand was lost. There were numerous stages at which the error could have been recognised, specifically: at the stage of retrieving and handing out the specimen; including the gallbladder bag in the scrub count; checking the specimen into the pot; and sending the empty specimen pot to pathology. This is a classic example illustrating Reason's 'Swiss cheese' model, where the holes line up to allow a hazard to be converted into an accident. ${ }^{1}$ These stages have been scrutinised, and theatre staff have had training in new protocols to try and prevent a similar event from happening again. The patient was discharged that night and made a good recovery despite having had two anaesthetics.

The National Safety Standards for Invasive Procedures outline mandatory protocols to prevent retained foreign objects. $^{2}$

\section{References}

1. Reason J. Human Error. Cambridge: Cambridge University Press; 1990.

2. NHS England. National Safety Standards for Invasive Procedures (NatSSIPS). London: NHS England; 2015.

\section{Transanal troubles}

(Ref 229)

Transanal minimally invasive surgery (TAMIS) is a new technique to treat early rectal cancer and benign polyps in the rectum. A 75-year-old man with a past history of deep vein thrombosis (treated with warfarin) was found to have a high grade dysplastic rectal polyp on colonoscopy although histology was equivocal. Computed tomography (CT) and magnetic resonance imaging (MRI) were inconclusive, suggesting that this might be a T1 tumour. The patient underwent TAMIS, at which an R1 resection (with a positive margin leaving residual cancer) was performed, histology subsequently suggesting a more advanced tumour. An anterior resection was therefore undertaken, which was made complicated by scarring from previous surgery. The patient developed a leak and recurrence, with tumour seeding, requiring extralevator abdominoperineal excision.

\section{Reporter's comments}

The first biopsy suggested high grade dysplasia but provided an inadequate sample. CT and MRI were not conclusive for tumour staging on full-thickness TAMIS excision. In retrospect, it appeared that the stage was at least T2. Proceeding to anterior resection as an initial definitive treatment might have been more appropriate under these circumstances.

\section{CORESS comments}

This is a technical case in the specialty sphere of transanal surgery. The key to success in these procedures lies in accurate patient selection and staging. Preoperative staging is of paramount importance in decision making. Tumour biopsies have low accuracy and histological discrepancies are well recognised. Endorectal ultrasonography and MRI also have acknowledged interobserver variability. Digital examination may often provide the most helpful and reliable information. 